\title{
Padrão de oviposição e tabela de vida da traça-do-tomateiro Tuta absoluta (Meyrick) (Lepidoptera, Gelechiidae)
}

\author{
Maria Alice de Medeiros ${ }^{1}$, Edison Ryoiti Sujii ${ }^{2}$, Guilherme Côrrea Rasi ${ }^{3}$, Ronaldo Setti Liz ${ }^{1}$ \\ \& Helena Castanheira de Morais ${ }^{3}$
}

'Embrapa Hortaliças, Caixa Postal 218, 70359-970, Brasília-DF, Brasil. maria.alice@embrapa.br, setti@cnph.embrapa.br

${ }^{2}$ Embrapa Recursos Genéticos e Biotecnologia, Caixa Postal 2372, 70770-900, Brasília-DF, Brasil. sujii@ cenargen.embrapa.br

${ }_{3}^{3}$ Departamento de Ecologia, Universidade de Brasília, 70910-900, Brasília-DF, Brasil. guilherme_rasi@yahoo.com.br; morais@unb.br

\begin{abstract}
Oviposition pattern and life table of South American tomato pinworm Tuta absoluta (Meyrick) (Lepidoptera, Gelechiidae). The tomato pinworm presented higher oviposition when colonizing tomato plants cropped in conventional system than in organic system, in previous field studies. Aiming to confirm and to understand the observed pattern in the field, bioecological processes, such as oviposition and mortality of immatures, were compared on ploted plants in soil from organic and conventional systems in semi-controlled conditions of greenhouse. Tomato pinworm adults were released in the greenhouse, the infested plants were transfered to another greenhouse after $24 \mathrm{~h}$, to track horizontal cohort. Naturally deposited eggs were located, marked and the leaf was caged. Each plant was observed daily and development stage or death was registered until all individuals completed the life cycle or died. Oviposition by tomato pinworm was 2-fold higher in plants growing in soil from conventional system than from organic system. The survivorship curve of immatures and life table in the greenhouse showed that there was no difference in organic and conventional plants. Thus, the difference in the oviposition pattern observed in the field is not related to the progeny performance and can be influenced by the environment of organic tomato crop system.
\end{abstract}

KEYWORDS. Host selection; insect-plant interaction; nutritional ecology; survivorship.

RESUMO. Padrão de oviposição e tabela de vida da traça-do-tomateiro Tuta absoluta (Meyrick) (Lepidoptera, Gelechiidae). A traça-do-tomateiro apresentou maior oviposição ao colonizar tomateiros cultivados em sistema convencional em relação ao orgânico em estudos prévios realizados no campo. Visando confirmar e entender o padrão observado no campo, aspectos bioecológicos como oviposição e mortalidade de imaturos foram comparados em condições semicontroladas de casa de vegetação com plantas cultivadas em vasos com solo proveniente do sistema orgânico e convencional. Adultos da traça-do-tomateiro foram liberados na casa de vegetação e após $24 \mathrm{~h}$, as plantas infestadas foram transferidas para outra casa de vegetação, para acompanhamento de coortes horizontais. Os ovos naturalmente depositados pela traça-do-tomateiro foram localizados na planta e demarcados. Em seguida, a folha foi ensacada. Diariamente as plantas foram observadas, registrando a fase de desenvolvimento do inseto e a ocorrência de morte, até que todos os insetos completassem seu ciclo de vida. A oviposição pela traça-do-tomateiro em plantas com solos oriundos do sistema convencional foi duas vezes maior do que em plantas com solos do sistema orgânico. A curva de sobrevivência da fase imatura e a tabela de vida da traça-do-tomateiro em casa de vegetação mostraram que a sobrevivência em plantas com solo orgânico e convencional não apresentaram diferenças. Assim as diferenças no comportamento de padrão de oviposição observadas a campo provavelmente não estão relacionadas com o desempenho da progênie e podem ser influenciado pelo ambiente proporcionado pelo sistema orgânico de produção.

PALAVRAS-CHAVE. Ecologia nutricional; interação inseto-planta; seleção do hospedeiro; sobrevivência.

O crescimento, desenvolvimento e reprodução dos insetos dependem diretamente da qualidade e quantidade do alimento utilizado (Hagen et al. 1984). Insetos herbívoros normalmente selecionam as plantas de acordo com o seu conteúdo nutricional. Dessa forma, dependem das plantas para sobreviver e estão sujeitos a todas as mudanças resultantes das interações entre as plantas e o ambiente (Pizzamiglio 1991). A susceptibilidade da planta a um ataque de insetos depende não somente de suas características químicas e morfológicas, mas também do meio ecológico. Por exemplo, se a planta cresce em uma comunidade que sustenta uma população de insetos predadores, essa associação confere proteção para a planta. Do mesmo modo, a presença de substâncias repelentes liberadas no ar por outras espécies de plantas também confere proteção (Edwards \& Wratten 1981).
A agricultura orgânica baseia-se no uso de fertilizantes orgânicos, complementados por adubos minerais pouco solúveis, sendo capaz de fornecer todos os macros e micronutrientes de que as culturas necessitam em doses proporcionais, tornando-as mais resistentes à ação das espécies prejudiciais (Paschoal 1994). A agricultura convencional, por sua vez, utiliza intensivamente fertilizantes inorgânicos altamente solúveis e controle químico de pragas. A adoção de práticas mais sustentáveis ao agroecossistema somente será possível pela comparação entre os sistemas de baixo insumo e o convencional e o entendimento dos mecanismos de interações ecológicas envolvidas (Betiol et al. 2004). A cultura do tomate, devido principalmente ao uso intenso de insumos agroquímicos e o alto risco de contaminação dos consumidores, produtores e ambiente, é 
um excelente modelo para fazer comparações entre os sistemas orgânico e convencional de produção (Drinkwater et al. 1995).

A traça-do-tomateiro, Tuta absoluta (Meyrick) (Lepidoptera: Gelechiidae) é um dos principais insetos-praga do tomateiro. As larvas minam as folhas, broqueiam o caule, perfuram o broto terminal e atacam os frutos, principalmente, na região de inserção do cálice, onde encontram apoio para penetrar. O ciclo completo da traça-do-tomateiro dura de 26 a 30 dias. O estágio de ovo dura de três a seis dias, sendo que $77 \%$ das larvas eclodem entre o quarto e quinto dia (Coelho \& França 1987). O estágio de larva, que é o causador dos danos, dura 14 dias. O estágio de pupa dura cerca de oito dias e pode se desenvolver dentro da própria lesão ou no solo. Após o estágio de pupa, os adultos emergem. Cada fêmea pode depositar de 55 a 130 ovos durante três a sete dias (Coelho \& França 1987; Haji et al. 1988). Existe uma maior concentração de posturas no terço superior das plantas, sendo que a maioria dos ovos é depositada nas folhas (Haji et al. 1988; Torres et al. 2001; Pratissoli et al. 2003).

Considerando que a traça-do-tomateiro é um inseto-praga com alta capacidade reprodutiva e específica do tomateiro, muitos trabalhos sobre a sua biologia já foram publicados (Coelho \& França 1987; Haji et al. 1988; Imenes et al. 1990; Bentancourt et al. 1996; Mihsfeldt \& Parra 1999; GonçalvesGervásio et al. 1999; Thomazini et al. 2001; Pereyra \& Sánchez 2006). Entretanto dentre estes trabalhos poucos são relacionados com a sua ecologia. Uma exceção é o trabalho de Miranda et al. (1998) que elaborou uma tabela de vida ecológica para a traça-do-tomateiro no campo. Nesse sentido, Southwood (1978) relatou que a construção das tabelas de vida é importante para o entendimento da dinâmica populacional de uma espécie.

A colonização da traça-do-tomateiro foi comparada em sistema de produção orgânico e convencional, onde se verifica que, embora o tamanho das populações circundantes à área do experimento não apresentasse diferenças significativas, o comportamento de colonização das plantas pelo inseto foi diferente dentro das áreas experimentais, sendo que o sistema convencional apresentou maiores densidades de ovos da traça-do-tomateiro (Medeiros 2007). O objetivo deste trabalho foi avaliar o padrão de oviposição da traça-do-tomateiro em condições semi-controladas em plantas cultivadas com solo provenientes do sistema convencional e orgânico e verificar se esse padrão está relacionado ao desempenho da progênie com reflexos na sua dinâmica populacional.

\section{MATERIALE MÉTODOS}

\section{Área do Experimento e Criação da traça-do-tomateiro.}

Os experimentos foram conduzidos no campo experimental da Embrapa Hortaliças, Brasília-DF, localizado a latitude $15^{\circ} 56^{\prime} \mathrm{S}$, longitude $48^{\circ} 08^{\prime} \mathrm{W}$ e altitude $997,6 \mathrm{~m}$, em casa de vegetação, de abril a junho de 2006.

Para este estudo foi necessário estabelecer uma criação de traça-do-tomateiro. Adultos foram coletados em Ponte Alta,
Brazlândia e Vargem Bonita, DF. Ramos de tomateiros com folhas verdes e infestadas pela traça foram coletados em lavouras em fase de colheita. As folhas de tomateiro infestadas foram colocadas em sacos de papel pardo que foram lacrados com grampos, para induzir a empupação das larvas. Após cinco dias os sacos foram abertos e as pupas coletadas e mantidas em câmaras climatizadas tipo BOD, a $25 \pm 2^{\circ} \mathrm{C}, 60 \pm 10 \%$ UR e fotofase de $10 \mathrm{~h}$. Quando os adultos começaram a emergir foram transferidos para uma casa de vegetação, contendo vasos com tomateiros para manutenção e multiplicação dos insetos.

\section{Solo para a execução dos experimentos.}

Quantidades de solos foram coletados em área de latossolo vermelho escuro no campo experimental da Embrapa Hortaliças, em área destinada ao sistema de produção orgânico que se encontra sob este manejo desde 2001 e em área destinada ao sistema de produção convencional. Amostras destes solos foram tomadas para a análise química no Laboratório de solos da Embrapa Hortaliças. Os solos coletados foram colocados em vasos com capacidade de 2,01.

Estudo do padrão de oviposição.

Em casa de vegetação os vasos com plantas de tomateiro cultivar Duradoro em floração, foram colocados sobre as bancadas, dispostos em conjuntos com quatro vasos distribuídos ao acaso, totalizando 20 conjuntos: 10 conjuntos com solos oriundos de sistema orgânico e 10 conjuntos com solos oriundos de sistema convencional, em posição previamente sorteada. Em seguida, 80 adultos da traça-dotomateiro em placas de Petri (quatro indivíduos para cada conjunto) foram liberados de forma equiitativa. Após $24 \mathrm{~h}$, as plantas foram observadas, sendo que as plantas infestadas foram transferidas para outra casa de vegetação para acompanhamento diário do ciclo biológico. A análise foliar das plantas infestadas (três amostras) e não infestadas (uma amostra) pela traça-do-tomateiro foi feita pelo Laboratório de Química do Solo da Embrapa Hortaliças.

Tabela de vida da traça-do-tomateiro em casa de vegetação.

Os ovos naturalmente depositados pela traça-do-tomateiro foram localizados na planta e demarcados com círculo, no folíolo, com caneta hidrográfica de cor preta. Em seguida, a folha foi protegida com saco de tecido (de malha fina) que impedia a saída dos insetos. Diariamente as plantas foram observadas, registrando a fase de desenvolvimento do inseto e a ocorrência de morte, até que todos os insetos completassem seu ciclo de vida ou estivessem mortos. Os dados de oviposição foram analisados estatisticamente pelo Teste quiquadrado com auxílio do programa SigmaStat 3.1 (Systat Software Inc. 2004), a elaboração da tabela de vida e a comparação das curvas de sobrevivência foram feitas com o programa Ecological Methodology (Krebs 1998). 


\section{RESULTADOS EDISCUSSÃO}

A oviposição pela traça-do-tomateiro, nas plantas cultivadas em solos provenientes do sistema convencional, foi duas vezes maior do que naquelas nos solos do sistema orgânico (Fig. 1), mostrando que a traça-do-tomateiro deposita mais ovos em plantas cultivadas no sistema convencional de produção. Esta observação comprova as observações realizadas em campo durante os anos de 2004 e 2005 (Medeiros 2007), em que a taxa de oviposição da traça-do-tomateiro foi duas e três vezes maior, respectivamente, em 2004 e 2005 no sistema convencional de produção, embora as populações da traça-do-tomateiro nas duas áreas experimentais avaliadas por meio de armadilhas de feromônio tenham sido semelhantes. Com base nesses resultados, pode-se inferir que existe alguma característica da planta, provavelmente bioquímica, que inibe a oviposição pela traça-do-tomateiro no sistema orgânico ou estimula a oviposição em sistema convencional de cultivo.

Dentre os elementos constituintes das plantas, o nitrogênio parece ser o mais importante do ponto de vista nutricional para os insetos. De acordo com Parra (1991), o nitrogênio tem um papel fundamental em todos os processos metabólicos e na codificação genética. Dentre os componentes alimentares é o nitrogênio, em termos de quantidade e qualidade disponíveis, o que geralmente limita o crescimento e a fecundidade de insetos. Em geral, insetos herbívoros especialistas predominam em plantas com alto teor de nitrogênio (McNeil \& Prestidge 1982) embora as relações específicas entre as espécies e os diversos elementos ainda não estejam esclarecidas. É fato que a aplicação de fertilizantes na agricultura, visando o aumento de rendimento das culturas pode aumentar ou diminuir os problemas associados com os herbívoros (Panizzi \& Parra 1991).

A interpretação da análise foliar das plantas nas quais a oviposição foi feita revelou que os índices de nitrogênio, fósforo e potássio foram maiores nas plantas cultivadas em solos provenientes do sistema de produção orgânico (Tabela

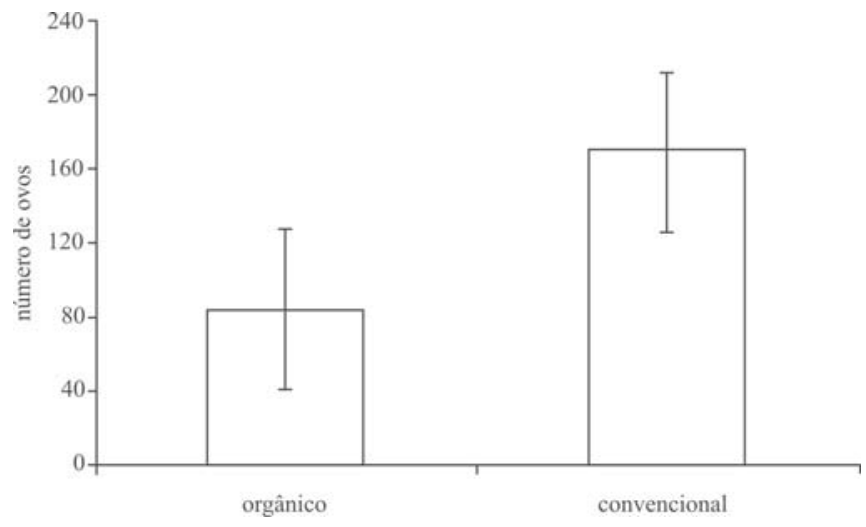

Fig. 1. Padrão de oviposição de Tuta absoluta em tomateiros plantados em solos provenientes de sistema orgânico $(n=30$, para 40 plantas disponíveis) e convencional ( $\mathrm{n}=35$, para 40 plantas disponíveis) em casa de vegetação. $\left(\chi^{2}=77,786\right.$, g.l. $\left.=38, P<0,001\right)$. Embrapa Hortaliças, DF, 2006 .
Tabela I. Teores totais de nitrogênio, fósforo e potássio obtidos pela análise foliar dos tomateiros cultivados em solos provenientes de sistema orgânico e convencional de produção. Embrapa Hortaliças, DF, 2006.

\begin{tabular}{llll}
\hline Solo & $\mathrm{N} * *$ & $\mathrm{P}^{* *}$ & $\mathrm{~K} * *$ \\
\hline Convencional* & $34,05 \pm 0,64$ & $5,16 \pm 0,47$ & $19,64 \pm 1,42$ \\
Convencional não & & & \\
preferido & 46,28 & 6,52 & 21,22 \\
Orgânico* & $40,73 \pm 3,15$ & $8,69 \pm 1,47$ & $23,77 \pm 2,59$ \\
Orgânico não preferido & 52,82 & 11,74 & 21,75 \\
\hline
\end{tabular}

*valor referente à média entre três medidas

$* * \mathrm{~g} / \mathrm{kg}=\% \times 10$

I). Os valores encontrados para o teor de nitrogênio nas plantas preferidas e não-preferidas sugerem que deve existir um conteúdo ideal de nitrogênio para a espécie. $\mathrm{O}$ trabalho de Leite et al. (2003) em que os autores avaliaram o efeito da adubação sobre a incidência da traça-do-tomateiro mostrou que a maior percentagem de frutos atacados pela traça foi observada em plantas cultivadas sob o nível de adubação com maior teor de nitrogênio $\left(\mathrm{N}_{300} \mathrm{~K}_{0}\right)$. Letourneau et al. (1996) não encontraram indicações de que maiores teores de nitrogênio foliar em cultivo de tomate foi associado com maiores níveis de danos de insetos. De acordo com Altieri \& Nicholls (2003) são necessários maiores estudos sobre populações de insetos em plantas tratadas com fertlizantes orgânicos e com fertilizantes sintéticos. É importante ressaltar que podem existir outras explicações para o fato da traça-do-tomateiro depositar mais ovos em plantas do sistema convencional de produção como, por exemplo, a presença de substâncias secundárias, os teores de proteínas e aminoácidos totais, além de outros elementos nutricionais.

Um total de 24 adultos de traça-do-tomateiro foi obtido de 69 ovos, marcados em plantas cultivadas em solo proveniente do sistema orgânico, resultando em $65 \%$ de mortalidade (Tabela II), com $37 \%, 14 \%$ e $13 \%$ de mortalidade, respectivamente, nos estágios de ovo, larva e pupa. Um total de 39 adultos de traçado-tomateiro foi obtido de 107 ovos, marcados em plantas cultivadas em solo proveniente do sistema convencional,

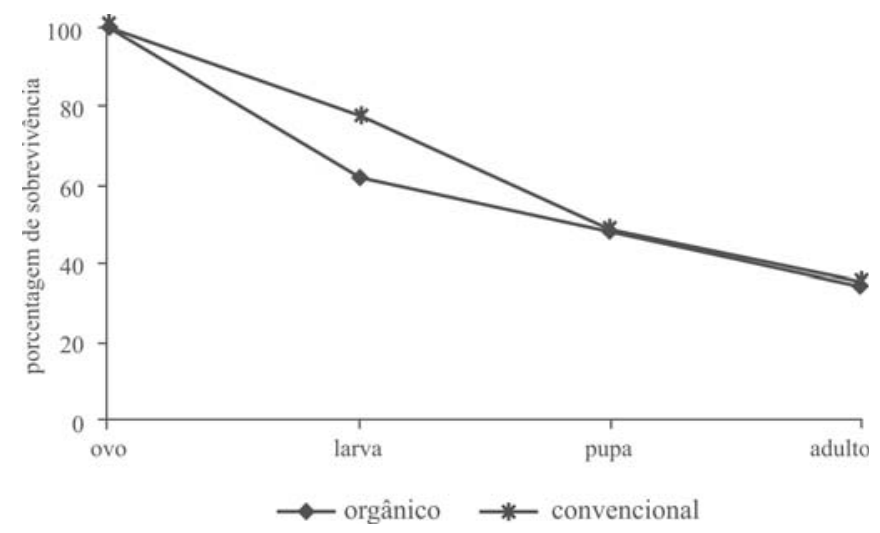

Fig. 2. Sobrevivência de Tuta absoluta em casa de vegetação, em plantas cultivadas em vasos com solos provenientes de sistema convencional e orgânico de produção. Embrapa Hortaliças, DF, 2006. 
Tabela II. Tabela de vida de Tuta absoluta em casa de vegetação com solos provenientes de sistema convencional e orgânico de produção. Embrapa Hortaliças, DF, 2006.

\begin{tabular}{llrcrcc}
\hline Tratamento & \multicolumn{1}{c}{$\chi$} & $n_{x}$ & $l_{x}$ & $d_{x}$ & $q_{x}$ & $l_{x}$ \\
\hline Convencional & Ovo & 107,0 & 1,0000 & 23,00 & 0,2150 & 1,0000 \\
& Larva & 84,0 & 0,7850 & 31,00 & 0,3690 & 0,7850 \\
& Pupa & 53,0 & 0,4953 & 14,00 & 0,2642 & 0,4953 \\
\multirow{4}{*}{ Orgânico } & Adulto & 39,0 & 0,3645 & 39,00 & 1,000 & 0,3645 \\
& Ovo & 69,0 & 1,0000 & 26,00 & 0,3768 & 1,0000 \\
& Larva & 43,0 & 0,6232 & 10,00 & 0,2326 & 0,6232 \\
& Pupa & 33,0 & 0,4783 & 9,00 & 0,2727 & 0,4783 \\
& Adulto & 24,0 & 0,3478 & 24,00 & 1,0000 & 0,3478 \\
\hline
\end{tabular}

$\chi=$ intervalo de idade

$\mathrm{n}_{\mathrm{x}}=$ número de indivíduos de uma coorte vivos no início de cada intervalo de idade

$1_{x}=$ proporção de indivíduos sobreviventes no início de cada intervalo de idade

$\mathrm{d}_{\mathrm{x}}=$ número de indivíduos de uma coorte que morreram durante o intervalo de idade $\mathrm{x}$ até $\mathrm{x}+1$

$\mathrm{q}_{\mathrm{x}}=$ taxa finita de mortalidade durante o intervalo de idade de $\mathrm{x}$ até $\mathrm{x}+1$ $\mathrm{p}_{\mathrm{x}}=$ taxa finita de sobrevivência durante o intervalo de idade de $\mathrm{x}$ até $\mathrm{x}+1$

resultando em $64 \%$ de mortalidade (Tabela II), com 21\%, 29\% e $13 \%$ de mortalidade, respectivamente, nos estágios de ovo, larva e pupa. A sobrevivência obtida nos dois tratamentos não foi significativamente diferente $\left(\chi^{2}=0,23, P>0,05\right)$, apesar dos tratamentos terem apresentado diferenças discretas nos estágios de ovo e larva. Com este resultado conclui-se que as diferenças no comportamento de colonização do tomateiro, nos dois sistemas de produção observadas no campo, provavelmente estão relacionadas com o ambiente, especialmente com o ambiente proporcionado pelo sistema orgânico de produção.

Uma redução linear da sobrevivência durante o período de ovo até adulto caracterizando uma curva do tipo 2 (Southwood 1978; Begon et al. 1996; Southwood \& Hendersen 2000) foi observada nos dois tratamentos (orgânico e convencional) (Figura 2). Foi observada uma curva semelhante no campo, para o estágio de ovo. Como o experimento foi conduzido em casa de vegetação, fatores de mortalidade como predadores e parasitóides e ação mecânica da água/chuva que já foram indicados em outros trabalhos como importantes (Miranda et al. 1998; Medeiros 2007) foram excluídos. Dessa forma, foi evidenciada a mortalidade intrínseca que ocorre na população em condições semicontroladas. Para o estágio de ovo, tanto no tratamento convencional quanto no orgânico, a causa principal de mortalidade foi a inviabilidade de ovos, para os estágios de larva e pupa é provável que o fator de maior influência seja relacionado às variações nutricionais. Miranda et al. (1998) determinaram no campo que a mortalidade da traçado-tomateiro foi de $92 \%$, com $58 \%$, 33\% e $0,68 \%$ de mortalidade nos estágios de ovo, larva e pupa, respectivamente. Neste mesmo trabalho foi observado que o estágio de larva foi mais crítico e que os predadores foram responsáveis por $99,5 \%$ da mortalidade, sendo que para o estágio de ovo a inviabilidade foi o principal fator de mortalidade.

Os resultados do presente trabalho confirmaram as observações de campo, em que a traça-do-tomateiro oviposita mais em plantas cultivadas sob o sistema de produção convencional (Medeiros 2007). Esse padrão não está relacionado a um melhor desempenho da progênie, já que em casa de vegetação observou-se que a sobrevivência não apresentou diferença nos dois tratamentos (traça-do-tomateiro criadas em plantas cultivadas em solo orgânico e convencional). Esses resultados estão de acordo com a teoria de preferência-desempenho proposta por Price et al. (1990), em que espécies eruptivas (pragas) apresentam baixa relação entre a preferência das fêmeas por plantas ou locais de oviposição e o desempenho da respectiva progênie, embora não expliquem a causa dessa preferência. A atratividade de plantas cultivadas em sistemas orgânicos aos predadores e parasitóides, através da emissão de semioquímicos, é uma possível explicação para esse fenômeno de preferência destes grupos de artrópodes na cultura embora não tenham sido quantificados. As populações menores de traça-do-tomateiro observados em campos cultivados no sistema orgânico, devem-se a uma combinação de não-preferência de oviposição associada a maior quantidade de predadores generalistas afetando a sobrevivência da progênie (Medeiros 2007).

Agradecimentos. À Dra. Geni L. Villas Bôas e aos revisores anônimos pelas contribuições no manuscrito e ao Dr. André N. Dusi pelo empréstimo da casa de vegetação para execução do experimento. Este trabalho faz parte da tese de doutorado desenvolvida pela autora senior (MAM) no programa de pós-graduação em Ecologia da Universidade de Brasília.

\section{REFERÊNCIAS}

Altieri, M. A. \& C. I. Nicholls. 2003. Soil fertility management and insect pest: harmonizing soil and plant health in agroecosystems. Soil \& tillage research 72: 203-211.

Begon, M.; J. L. Harper \& C. R. Townsend. 1996. Ecology: individuals, populations and communities. 3rd. ed. London, Blackwell Scientific Publications, 1068 p.

Bentancourt, C. M.; I. B. Scatoni \& J. J. Rodríguez. 1996. Influencia de la temperatura sobre la reproducción y el desarrollo de Scrobipalpuloides absoluta (Meyrick) (Lepidoptera, Gelechiidae). Revista Brasileira Biologia 56: 661-670.

Betiol, W.; R. Ghini; J. A. H. Galvão \& R. C. Siloto. 2004. Organic and conventional tomato cropping systems. Scientia Agricola 61: $253-259$.

Coelho, M. C. F. \& F. H. França. 1987. Biologia, quetotaxia da larva e descrição da pupa e adulto da traça-do-tomateiro. Pesquisa Agropecuária Brasileira 22: 129-135.

Drinkwater, L. E.; D. K. Letourneau; F. Workneh; A. H. C. V. Bruggen \& C. Shennan. 1995. Fundamental differences between conventional and organic tomato agroecosystems in California. Ecology Applied 5: $1098-1112$.

Edwards, P. J. \& S. D. Wratten. 1981. Ecologia das interações entre insetos e plantas. São Paulo, EDUSP, 71 p.

Gonçalves-Gervásio, R. C. R.; A. I. Ciociola; L. V. C. Santa-Cecília \& W. R. Maluf. 1999. Aspectos biológicos de Tuta absoluta (Meyrick, 1917) (Lepidoptera: Gelechiidae) em dois genótipos de tomateiro contrastantes quanto ao teor de 2-tridecanona nos folíolos. Ciencia Agrotecnologia 23: 247-251.

Hagen, K. S.; R. H. Dadd \& J. Reese. 1984. The food of insects. p.79112. In: Huffaker, C. B. \& R. L. Rabb (eds.), Ecological methodology. New York, J.Wiley \& Sons, 844 p.

Haji, F. N. P. ; J. R. P. Parra; J. P. Silva \& J. G. S. Batista. 1988. Biologia 
da traça do tomateiro sob condições de laboratório. Pesquisa Agropecuária Brasileira 23: 107-110.

Imenes, S. D. L.; M. A. Uchôa-Fernandes; T. B. Campos \& A. P. Takematsu. 1990. Aspectos biológicos e comportamentais da traçado-tomateiro Scrobipalpula absoluta (Meyrick, 1917), (Lepidoptera-Gelechiidae). Arquivos Instituto Biológico 57: 6368.

Krebs, C. J. 1998. Ecological methodology, 2nd ed., California, Addison Wesley Longman, $620 \mathrm{p}$.

Leite, G. L. D.; C. A. Costa; C. I. M. Almeida \& M. Picanço. 2003. Efeito da adubação sobre a incidência de traça-do-tomateiro e Alternaria sp. em plantas de tomate. Horticultura Brasileira 21: $448-451$.

Letourneau, D. K.; L. E. Drinkwater \& C. Shennan. 1996. Effects of soil mamagement on crop nitrogen and insect damage in organic vs. conventional tomato fields. Agriculture, Ecosystems \& Environment 57: 179-187.

McNeil, S. \& R. A. Prestidge. 1982. Plant nutricional strategies and insect herbivore community dynamics, p. 225-235. Proc. $5^{\text {th }}$ Int. Symp. Insect-Plant Relationships, Wageningen.

Medeiros, M. A. 2007. O papel da biodiversidade no manejo da traçado-tomateiro Tuta absoluta (Meyrick, 1917) (Lepidoptera: Gelechiidae). Tese de Doutorado, Universidade de Brasília. 145 p.

Mihsfeldt, L. H. \& J. R. P. Parra. 1999. Biologia de Tuta absoluta (Meyrick, 1917) em dieta artificial. Scientia agricola 56: 769776.

Miranda, M. M. M.; M. Picanço; J. C. Zanúncio \& R. N. C. Guedes. 1998. Ecological life table of Tuta absoluta (Meyrick) (Lepidoptera: Gelechiidae). Biocontrol Sciencia Technology 8: 597-606.

Panizzi, A. R. \& J. R. R. Parra. 1991. A ecologia nutricional e o manejo integrado de pragas. p. 313-336. In: A. R. Panizzi \& J. R. P. Parra (eds.), Ecologia nutricional de insetos e suas implicações no manejo de pragas. São Paulo, Manole, $412 \mathrm{p}$.

Parra, J. R. P. 1991. Consumo e utilização de alimentos por insetos p. 9-65. In: A. R. Panizzi \& J. R. P. Parra (eds.), Ecologia nutricional de insetos e suas implicações no manejo de pragas. São Paulo, Manole, $412 \mathrm{p}$.
Paschoal, A. D. 1994. Produção orgânica de alimentos: agricultura sustentável para os séculos XX e XXI, Piracicaba, $191 \mathrm{p}$.

Pereyra, P. C. \& N. E. Sánchez. 2006. Effect of two solanaceous plants on development and population parameters of the tomato leaf miner, Tuta absoluta (Meyrick) (Lepidoptera: Gelechiidae). Neotropical Entomology 35: 671-675.

Pizzamiglio, J. R. P. 1991. Ecologia das interações entre inseto/planta. p. 101-129. In: A. R. Panizzi \& J. R. P. Parra (eds.), Ecologia nutricional de insetos e suas implicações no manejo de pragas. São Paulo, Manole, $412 \mathrm{p}$.

Pratissoli, D.; J. R. P. Parra; O. A. Fernandes; R. C. Oliveira; H. B. Zago \& F. F. Pereira. 2003. Oviposition pattern of the tomato leafminer Tuta absoluta (Lepidoptera: Gelechiidae) on tomato under different population densities in greenhouse. Agro-ciência 19: 11-15.

Price, P. W.; N. Cobb; T. Craig; W. Fernandes; J. Itami; S. Mopper \& R. Preszler. 1990. Insect herbivore population dynamics on trees and shrubs: new approaches relevant to latent and eruptive species. $p$ 1-38. In: E. A. Bernays (ed.), Insect-plant interactions. Florida, Boca Raton, CRS Press, v. 2. 208 p.

Systat Software Inc. 2004. SigmaStat 3.1 for Windows. INSO Corporation, Richmond, CA, USA, 848 p.

Southwood, T. R. E. 1978. Ecological methods with particular references to the study of insect populations. 2nd. ed., New York: Chapman \& Hall, 524 p.

Southwood T. R. E. \& P. A. Hendersen. 2000. Ecological methods 3rd. ed., Blackwell Science. 575 p.

Thomazini, A. P. B. W.; J. D. Vendramin; R. Brunherotto \& M. T. R. Lopes. 2001. Efeito de genótipos de tomateiro sobre a biologia e oviposição de Tuta absoluta (Meyrick) (Lep.: Gelechiidae). Neotropical Entomology 30: 283-288.

Torres, J. B.; C. A. Faria; W. S. Evangelista \& D. Pratissoli. 2001 Within-plant distribution of the leaf miner Tuta absoluta (Meyrick) immatures in processing tomatoes, with notes on plant phenology. Integrated Journal Pest Management 47: 173-178. 